\title{
Weather-Routing System Based on METOC Navigation Risk Assessment
}

\author{
Tommaso Fabbri * (D) and Raul Vicen-Bueno \\ NATO STO Centre for Maritime Research \& Experimentation (CMRE), 19126 La Spezia, Italy; \\ raul.vicen@cmre.nato.int \\ * Correspondence: tommaso.fabbri@cmre.nato.int; Tel.: +39-0187-527-349
}

Received: 26 March 2019 ; Accepted: 25 April 2019 ; Published: 2 May 2019

\begin{abstract}
This paper presents a novel weather-routing system based on a multi-criteria setup. The set of 3 conflicting criteria is: travel time, ship navigation added resistance (caused by wind and waves), and navigation risk/safety. To this aim, the International Maritime Organization (IMO) safety guidelines are exploited for the design of navigation risk criterion as a function of the METeorological and OCeanographic (METOC) and sailing conditions. This risk is directly integrated into the multi-criteria setup, as an innovative alternative to the systems proposed in the open literature. The proposed methodology is tested in a real operational scenario in the Mediterranean Sea. The obtained results show how the proposed system provides alternative routes with minimum risk to the decision-makers, as well as other different alternative routes minimizing the other criteria.
\end{abstract}

Keywords: weather routing; decision support; maritime risk assessment; maritime safety; pareto front; METOC

\section{Introduction}

In the last decade, the research field of weather routing has gained attention within naval operations as a decision-making tool to increase safety at sea, operational endurance, and reduce greenhouse gas (GHG) emissions [1]. A proper operational planning and decision-making methodology can achieve $2-4 \%$ in reduction of GHG emissions and therefore savings in fuel consumption [1]. To this aim, the development of a weather-routing system facilitates the efficient planning of the route toward a selected destination. This is achieved by taking into account METOC forecasts, the derived sailing conditions based on the vessel type and the selected ship operational state (speed, heading, loading conditions, etc.). Given the nature of the problem, the weather-routing problem is usually addressed in an optimization setup. The effectiveness of the provided route not only depends on the quality and accuracy of the METOC forecasts or ship hydrodynamic model but also on the modeling of the optimization problem and the selected methodology to solve it. Several approaches were developed to model the weather-routing problem. They go from methods in optimal control theory [2], dynamic programming to constrained graph problems [3,4], constrained nonlinear optimization problem [5] to a combination of them [6]. These mathematical methods are used either in a single-objective or multi-objective optimization setup.

Depending on the requirements of the ship's operator or the operational scenario, the objective of the weather routing can shift from one metric to another. The main objectives are usually focused on optimizing the voyage based on energy efficiency or travel time while providing solutions to the decision-makers as fast as possible. This kind of setup may also include a set of requirements to be satisfied as a minimum, including flexible or fixed arrival time. Besides time constraints, additional restrictions could also be considered. As an example, the significant wave height operational limits or critical encounter periods for avoiding dangerous situations could also be introduced. 
Reference systems in the open literature $[3,4,7]$ use the navigation safety guidelines [8] for the definition of navigation constraints, which limit the areas where the navigation is allowed and not allowed based on the METOC conditions and the derived navigation conditions. In contrast to these reference systems $[3,4,7]$ and similar to the approach discussed in $[9,10]$, the focus of our paper is on the translation of the navigation safety guidelines [8] into a safety criterion to be minimized within a multi-criteria optimization setup. This design choice is based on the fact that the navigation safety of a route is usually determined by the vessel operator during the decisional stage. For this reason, we consider a conservative and limiting approach the removal of areas where the navigation may be too risky or not safe based on ad-hoc constraints which may produce a reduced solution space. For the above-mentioned considerations, this paper proposes a weather-routing system where the navigation safety represents a criterion to be minimized together with other additional criteria, such as the travel time and the added resistance caused by the interaction of the ship with the marine environment (e.g., winds and sea waves).

The proposed system tries to overcome the limitations faced by the operator during decisional stage. In contrast to reference systems $[7,9]$ where a multi-criteria ranking method is applied to sort the set of routes according to operator preferences and select the most suitable route, the proposed weather-routing system presents an alternative in the way it provides the results to the operator. The results are presented in the form of a reduced set of Pareto optimal routes. These are identified through a novel methodology exploiting the Hyper Radial Visualization (HRV) approach to explore the decision space by the introduced safety navigation criterion. This allows: first, the clustering of the routes based on predetermined navigation safety threshold, and second, the identification of the most suitable route in terms of travel time and added resistance for each navigation safety threshold. The availability of a reduced set of Pareto optimal routes allows the operator to avoid the analysis and the selection of a solution (route) among all the ones available. Therefore, compared with the reference systems, the proposed solution presents the advantage of untying the operator from the use of the only route provided as output. Finally, the availability of the reduced set of Pareto optimal routes allows the integration of the operator experience in the post analysis of the results e.g., selection of the candidate route based on additional requirements as the timing constraints or the operational scenario.

The paper is organized as follows. Section 2 presents the architecture of the proposed weather-routing system, including details about the proposed implementation. Section 3 presents the real scenario and the results for finding the best trade-off route between the two selected locations. Finally, the summary of the main findings and some ideas for future work are given in Section 4.

\section{Multi-Criteria Weather-Routing Framework}

Most of the available weather-routing systems are defined by an underlying common architecture of 4 interconnected components [1,4]: Environmental layer, Ship modeling, Planning layer and Decision layer. Each component provides a set of functionalities and data required for the complete setup of a weather-routing problem. An overview of the common architecture and its 4 components in the proposed system are depicted in Figure 1.

The Environmental layer provides the METOC information for the required Area of Interest (AOI) based on the defined time-frame (operator input requirements). To this aim, this component interfaces with remote data providers [11] or local databases to retrieve the required information. The Ship modeling describes the static and dynamic behavior with the aim of modeling and predicting the navigation status of the selected ship. Based on the data provided by the above-mentioned components, the Planning layer searches for the optimal route or the set of Pareto optimal solutions in the selected scenario. Finally, at the Decision layer, the decision-maker interacts with the system to select the final route. In the following subsections, each component of the underlying architecture is detailed. 


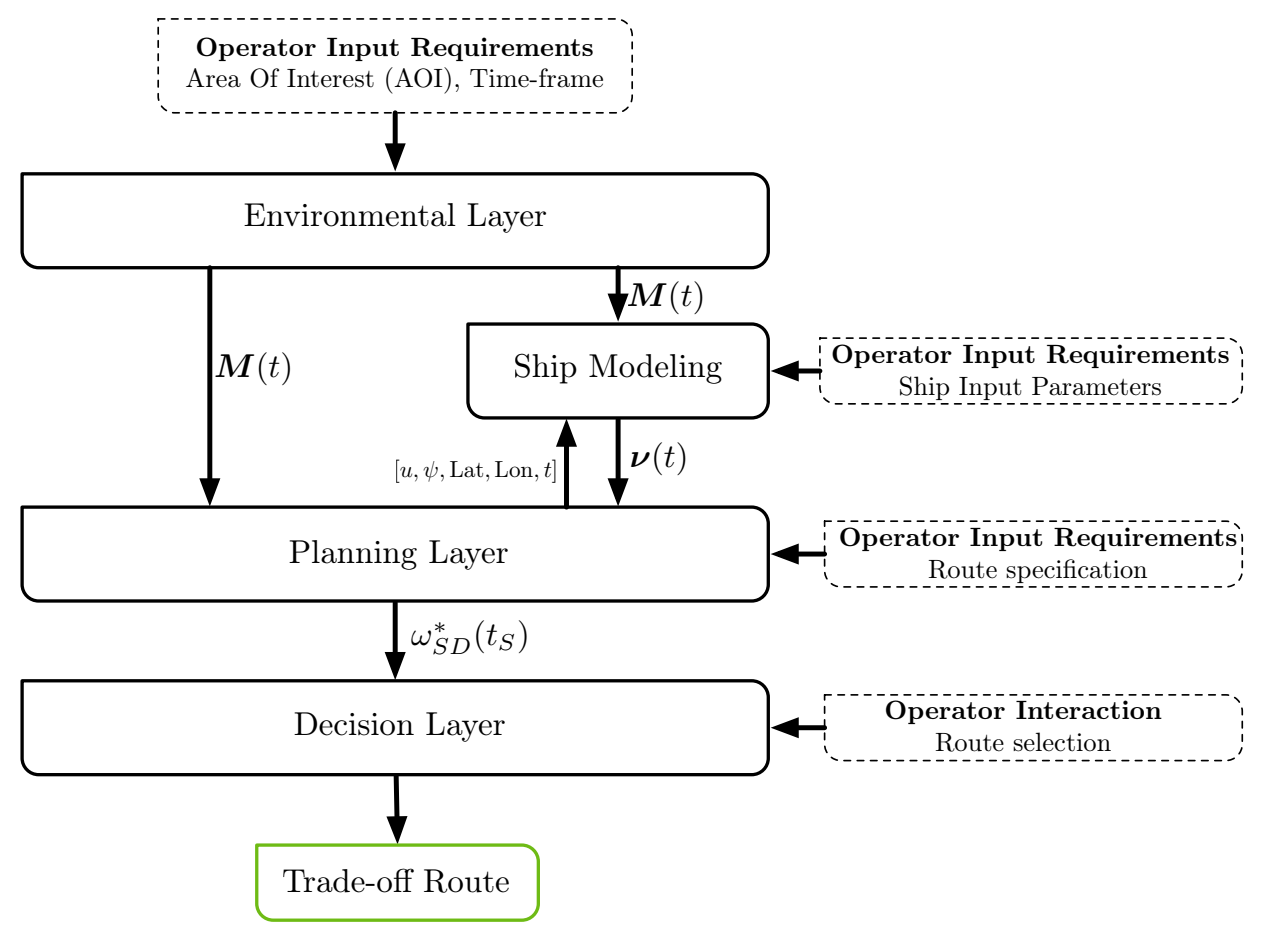

Figure 1. Underlying common architecture characterizing most of the available weather-routing systems.

\subsection{Environmental Layer}

The environmental layer is the system component managing the access to METOC data for the selected AOI. The data come in the form of field measurements (e.g., from satellites, sensor buoys, etc.) or as METOC forecasts. The availability and exploitation of these data provide knowledge of the current and predicted environmental state. Recalling the application domain of ship weather routing, the environmental layer considers the METOC variables/parameters having a direct impact on the navigation behavior and safety of a selected ship $[8,12]$. These METOC parameters are the ones able to characterize the waves of an irregular sea: significant wave height $\left(H_{S}[\mathrm{~m}]\right)$, peak wave period ( $T[\mathrm{~s}])$, wavelength $(\lambda[\mathrm{m}])$ and wave direction $\left(\alpha\left[^{\circ}\right]\right.$ respect to North direction - in this paper, we refer to the Geographic true North direction), as well as the wind through the mean wind direction $\left(\phi^{\circ}\right.$ respect to North direction) and the wind intensity/speed $\left(U_{10}\left[\mathrm{~m} \mathrm{~s}^{-1}\right]\right.$, measured at $10 \mathrm{~m}$ above the sea-surface). These METOC variables are used in the proposed system. Finally, the bathymetry or depth profile $(d[\mathrm{~m}])$ over the selected AOI is also used in the proposed system to identify the areas where ship navigation and maneuverability are limited or not allowed (due to the shallow water effect [13] or not enough under keel clearance). The availability of high-resolution bathymetry data allows us the estimation of more accurate $\lambda$ when this is not available from the local or remote provider. The deep-water approximation $\left(\lambda=\frac{g}{2 \pi} T^{2}\right.$, where $g$ is the standard gravitational acceleration) tends to overestimate the wavelength $\lambda$ in shallow water areas. The proposed framework uses the Hunt's method [14] characterized by an approximation error of the order of $0.1 \%$ in the entire range of depth values. Furthermore, the bathymetry represents an important parameter for the identification of the shallow water areas characterized by reduced maneuverability [13].

Starting from the operator requirements (AOI limits and selected time-frame), the environmental layer provides all the data to the underlying components (Ship modeling and Planning layer). The 7 environmental variables are all collected in a multi-dimensional grid $\boldsymbol{M}(t)$ as shown in Equation (1),

$$
\boldsymbol{M}(t)=\left[\boldsymbol{H}_{\boldsymbol{S}}(t), \boldsymbol{T}(t), \lambda(t), \boldsymbol{\alpha}(t), \boldsymbol{U}_{\mathbf{1 0}}(t), \boldsymbol{\phi}(t), \boldsymbol{d}\right] \in \mathbb{R}^{7 \times G_{\text {Lon }} \times G_{\text {Lat }}}
$$

where $t$ is a given time step, and $G_{\text {Lon }}$ and $G_{\text {Lat }}$ are the grid dimensions in the longitudinal and latitudinal directions, respectively. 


\subsection{Ship Modeling}

The Ship modeling is the system component in charge of the computation of the set of quantities/parameters describing the ship behavior while navigating. This component supports the Planning layer in the estimation of the optimal route or the set of routes toward a defined destination. The required parameters allow the identification of the sailing conditions for the selected ship based on the faced METOC conditions (provided by the environmental layer) and the estimated actual ship course (speed and heading) for a given [Lat, Lon] location at the time step $t$.

In this component, the shape of the ship and its hull is determined through a set of static parameters. These are the ship length, draft, beam width, metacentric height, and displacement. The navigation state is determined from the parameters defining the desired course. In this component, the navigation state is defined by the commanded speed $u[\mathrm{kn}]$ and heading $\psi\left[{ }^{\circ}\right.$ respect to North direction]. The complete set of parameters used in the proposed system is listed in Table 1, with typical values for a multi-role patrol frigate.

Table 1. Ship and navigation parameters used in the ship-modeling component and values for a typical multi-role patrol frigate.

\begin{tabular}{clccc}
\hline & Property & Symbol & Units & Value \\
\hline \multirow{5}{*}{ Ship parameters } & Length & $L_{S}$ & $\mathrm{~m}$ & 134.0 \\
& Beam width & $B$ & $\mathrm{~m}$ & 16.0 \\
& Draft & $T_{S}$ & $\mathrm{~m}$ & 5.0 \\
& Max Speed & $\bar{u}$ & $\mathrm{kn}$ & 35.0 \\
& Metacentric height & $G M$ & $\mathrm{~m}$ & 1.6 \\
& Displacement & $\Delta$ & $\mathrm{kg}$ & $4650 \times 10^{3}$ \\
\hline \multirow{2}{*}{ Navigation parameters } & Speed & $u$ & $\mathrm{kn}$ & {$[0, \bar{u}]$} \\
& Heading & $\psi$ & $\circ$ respect to N & {$[-\pi, \pi]$} \\
\hline
\end{tabular}

The desired accuracy determines the modeling aspects that may be included to estimate and predict the performance of the ship, such as hydrodynamic resistance, ship motions, comfort assessment, or engine performance prediction. Based on the requirement of providing solutions to decision-makers as fast as possible, a simplified model [15] of a ship is selected, limiting the number of parameters as shown in Table 1.

In the proposed system, the ship-modeling component represents a computational capability available to the Planning layer. This system component estimates the ship's capabilities due to the various METOC conditions: displacements, velocities, and accelerations are predicted considering the case of a ship sailing at constant speed, given a desired heading (determined by the selected $e \in \omega$, as discussed in the Section 2.3). These data are the result of a query from the Planning layer to the ship-modeling component. Each query specifies to the ship-modeling component the desired speed $(u)$ and heading $(\psi)$ for the selected ship at the location (Lat, Lon) in the time $t$. Then, the ship-modeling component computes the sailing conditions vector $v(t)$ (due to impact of the environment) computes the actual speed, $\hat{u}(t)$, heading, $\hat{\psi}(t)$ and the wave encounter period, $T_{E}(t)$ (the wave period experienced by the ship [15] determined considering the environmental data $\boldsymbol{M}(t)$ in the queried location).

\subsection{Planning Layer}

The planning layer represents the computational core of the proposed weather-routing system. Based on the data provided by the environmental layer and ship-modeling components, the waypoints composing the optimal route (or set of routes) is (are) computed. In its process, the route is optimized based on the designed cost(s) by taking into account the METOC information, ship properties, and route specification. A wide range of modeling approaches have been developed with linked mathematical methods. Approaches to the problem include methods based on isochrones [16], optimal control 
theory [17] and dynamic programming [18]. Furthermore, constrained graph methods, such as the Dijkstra algorithm, have been applied to ship routing in the Mediterranean Sea [3], Baltic sea [19] and Indian Ocean [20]. More recently, an increasing number of multi-objective weather-routing solutions have been proposed. Among them, the evolutionary algorithms have been used [5-7,9]. A complete review of the above-mentioned single and multi-objective weather-routing approaches has been presented in [21].

In the proposed implementation the weather-routing problem is addressed and solved as a multi-objective optimization problem through the Martins labeling algorithm [22]. This algorithm represents a generalization of the well-known Dijkstra algorithm [23] to support a multi-objective setup. In our implementation, the Martins algorithm is implemented in MATLAB, with additional libraries developed in JAVA to improve the performance of the whole system. The selected method requires the building of a navigation graph based on the route specification (departure and destination locations and a nominal route provided by the operator as input) and the definition of the costs associated with each edge (seaway between two nodes) of the graph.

\section{Navigation Graph}

The navigation graph defines the domain of available routes from which determining the route representing the solution of the weather-routing problem. The navigation graph is defined as an ordered pair

$$
\begin{aligned}
\mathcal{G}=(\mathcal{N}, \mathcal{E}) \quad \mathcal{N} & =\{1, \ldots, n\} \quad \text { the finite set of location nodes [Lon, Lat] } \\
\mathcal{E} & \subseteq \mathcal{N} \times \mathcal{N} \quad \text { the finite set of linking edges }
\end{aligned}
$$

Each edge $e \in \mathcal{E}$ represents the unique great-circle route (or shortest route) between the two linked nodes. The navigation graph is built around a provided nominal route connecting the departure and destination locations. The nominal route represents the shortest seaway between the selected locations, considering additional factors such as existing shipping lanes and specific traffic separation scheme. In this implementation, we refer as the nominal route the one provided by the web-service in [24]. From this, the possible alternative routes between the two locations are explored.

The navigation graph is built through the definition of the set

$$
\mathcal{S}=\bigcup_{i=0}^{m} \mathcal{S}_{i}
$$

where each $\mathcal{S}_{i}$ is the so-called stage, a set of nodes with ad-hoc spatial properties. Defined $\mathcal{S}_{0}$ and $\mathcal{S}_{m}$ as the elementary sets composed of only the departure and destination nodes, respectively, the stages $\mathcal{S}_{i \neq 0, m}$ are added to the navigation graph as follows. From the departure node $\left(\mathcal{S}_{0}\right)$ toward the destination node $\left(\mathcal{S}_{m}\right)$, a new stage every $z$ nmi along the nominal route is added (i.e., $z$ is the spatial resolution along the nominal route). Each stage $\mathcal{S}_{i \neq 0, m}$ is a set of $q$ equidistant nodes ([Lon, Lat $]$ ) placed over the perpendicular direction of the nominal route. Defined $b$ the stage length, the stage spacing $\frac{b}{q}$ represents the minimum distance between 2 nodes belonging to the same stage $\mathcal{S}_{i \neq 0, m}$. Each node of a stage $\mathcal{S}_{i}$ is linked to each node of the next stage $\mathcal{S}_{i+1}$ through a directed edge. New stages and related edges are added to the navigation graph until the final stage $\mathcal{S}_{m}$ (or destination node) is reached. Finally, the resulting navigation graph is cleaned of the nodes and edges where the navigation is not allowed or not possible (e.g., reduced maneuverability in shallow waters, edge crossing land, etc.). Figure 2 illustrates the process for the construction of the navigation graph spatial grid around the nominal route (in blue color) with $m=3$ stages and $q=5$ nodes per stage. 


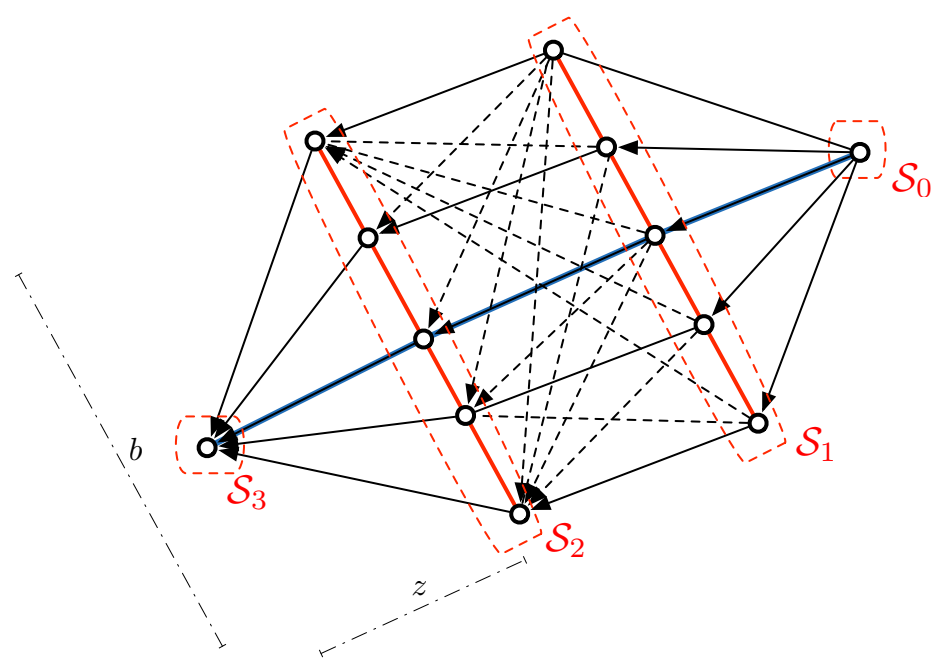

Figure 2. Example of navigation graph built around the nominal route in blue. This navigation graph is defined by $m=3$ stages with $q=5$ nodes per stage. The stages $\mathcal{S}_{0}$ and $\mathcal{S}_{3}$ are composed by only the departure and destination nodes, respectively.

The navigation graph requires the definition of the weight/s or cost/s associated with each edge for the computation of the optimal path between the departure and destination locations. In the proposed implementation, each edge $e \in \mathcal{E}$ has associated a set of 3 costs defining the costs for sailing through the selected edge. The 3 costs are collected in the vector

$$
c(e, t)=[\operatorname{tr}(e, t), r(e, t), n(e, t)]
$$

and detailed in the following paragraphs.

\section{Cost 1-Travel time}

The travel time $\operatorname{tr}(e, t)$ represents the estimate of the time required for traversing the edge $e \in \mathcal{E}$ based on the actual sailing conditions for the time step $t$. As defined in Equation (5), the travel time is computed based on the empirical model fully described in [25].

$$
\operatorname{tr}(e, t)=f_{t r}(e, t, \boldsymbol{M}(t), \boldsymbol{v}(t))
$$

This takes into account the average METOC conditions, $\boldsymbol{M}(t)$, and the estimated ship sailing conditions, $v(t)$, during the crossing of the edge $e$ to estimate the travel time $\operatorname{tr}(e, t)$. The implemented approach [25] assumes the ship moving between the consecutive node locations (or waypoints) with constant speed.

\section{Cost 2-Added resistance}

The ship total resistance, $R_{T}$, quantifies the ship losses (e.g., speed reduction) caused by the interaction of the ship with the waves for a given course. Several methodologies to estimate the resistance suffered by the ship characterized are available; each of these are characterized by different levels of accuracy [26]. In this paper, because high accuracy is not required, the estimation of ship total resistance is performed in two main steps following the approach defined in [27]:

- Estimate of the still/calm water resistance, $R_{S W}$, at the speed of interest.

- Estimate of the added resistance caused by the interaction of the ship with the waves, $R_{A W}$, at the speed of interest.

Following the approach developed in [27], the implemented criterion estimates the added resistance caused by the waves as the average percentage of speed loss $r(e, t)$ experienced by the 
ship on the edge $e \in \mathcal{E}$ at the time step $t$. This is done by considering the estimated sailing conditions, $v(t)$, for the selected ship as defined in Equation (6):

$$
r(e, t)=f_{r}(e, t, M(t), v(t))
$$

where $f_{r}$ is the empirical function defined in [27] estimating the contribution of added resistance. This criterion enables the user to investigate the relationship between fuel consumption and the various sea states and directions that the ship may encounter during the voyage [27]. The analysis of the fuel consumption and therefore the travel cost will be addressed in future works.

\section{Cost 3-Navigation risk}

The navigation risk criterion, $n(e, t)$, measures the average distance between the safe sailing conditions and the estimated sailing conditions faced by the selected ship when traveling through the edge $e \in \mathcal{E}$ at the time step $t$. This criterion is determined as function of the METOC state, as well as the ship parameters (e.g., length, beam width, displacement, etc. defined in Table 1), as follows in Equation (7).

$$
n(e, t)=f_{I M O}(e, t, \boldsymbol{M}(t), \boldsymbol{v}(t))
$$

The function $f_{I M O}$ is derived from the guidelines for navigators provided by the IMO [8]. This publication comprises a set of remarks and advices for the avoidance of dangerous states at sea. The dangerous phenomena considered in this work are:

- $\quad$ surf-riding and broaching-to;

- $\quad$ successive high-wave attack;

- synchronous rolling;

- reduction of intact-stability when riding a wave crest amidships.

Recalling the methodology proposed in $[9,10]$, the dangerous zones can be highlighted in the polar plot representation of Figure 3 for an exemplary case. This identifies the dangerous configurations of speed and course for a selected vessel: surf-riding and broaching-to in blue, synchronous rolling and parametric rolling in red and successive high-wave attack in green. Based on this representation the navigation risk criterion, $n(e, t)$, is related to the norm of the distance vector connecting the actual ship course (represented by the yellow marker in Figure 3) with the closest IMO dangerous phenomena, as shown in Figure 3 by the vector $f$. Defined as $\mathcal{D}_{I M O}(t)$ the set of ship course configurations within the IMO dangerous zones, the criterion is defined in Equation (9):

$$
\begin{aligned}
\bar{f} & =\min _{p \in \mathcal{D}_{I M O}(t)} \frac{\|v(t)-p\|}{\bar{u}} \\
n(e, t)=f_{I M O}(e, t, M(t), v(t)) & = \begin{cases}1-\bar{f} & \bar{f}<1 \\
0 & \text { otherwise }\end{cases}
\end{aligned}
$$

where $\bar{f}$ in Equation (8) represents the normalized distance. The criterion $n(e, t)$ has values in the range $[0,1]$, where 0 represents high distance from the dangerous phenomena (low risk) and 1 represents the state of high risk.

To complete the mathematical formalization of the weather-routing problem within the Planning layer, it is necessary to introduce the definition of a mathematical representation of a route between two end locations or nodes of the graph (graph walk). A walk $\omega$ in the graph $\mathcal{G}=(\mathcal{N}, \mathcal{E})$ is an ordered sequence of nodes and edges as follows 


$$
\omega=\left[n_{0}, e_{1}, n_{1}, \ldots, e_{\tau}, n_{\tau}\right]
$$

where each edge $e_{i}=\left(n_{i-1}, n_{i}\right)$ [28]. The length of the walk $\omega$ is $\tau$. It is worth pointing out that given the structure of the navigation graph composed by $m$ stages, the walks $\omega \in \Omega_{S D}$ connecting the departure $S$ and destination $D$ nodes are all characterized by the same length $\tau=m$.

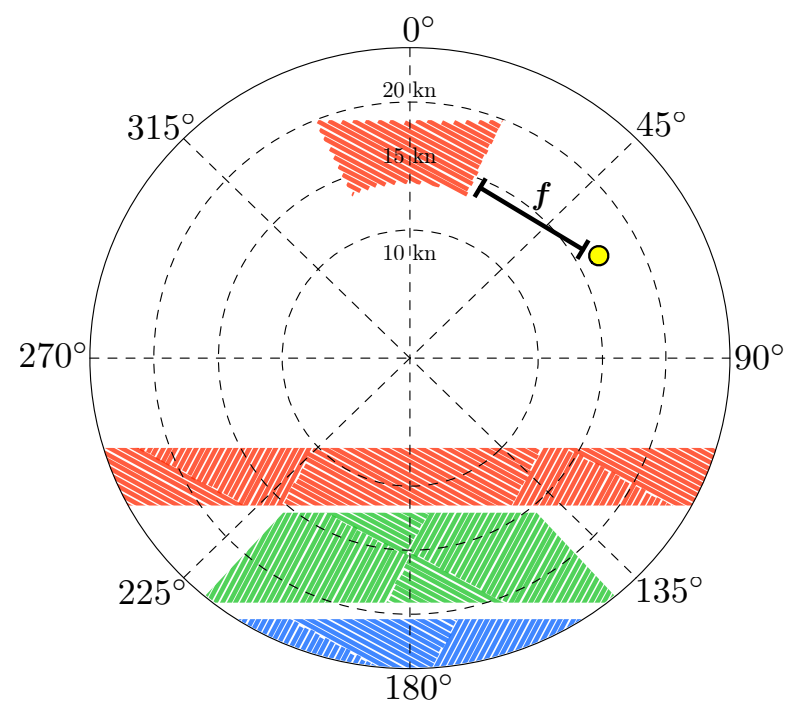

Figure 3. Representation of the dangerous zones according to the guidelines IMO [8]: surf-riding and broaching-to in blue, synchronous rolling and parametric rolling in red and successive high-wave attack in green.

Defined the navigation graph, $\mathcal{G}=(\mathcal{N}, \mathcal{E})$, and the vector of costs, $c(e, t)$, associated with the each edge $e$ at given time $t$, the aim of the weather-routing problem is to determine the optimal walk $\omega_{S D}^{*}\left(t_{S}\right) \in \Omega_{S D}$ connecting the departure node $S$ and the destination node $D$ considering a predefined departure time step $t_{S}$. This is achieved by solving a multi-objective optimization problem through the Martins algorithm [22] evaluating the cumulative costs of $\omega \in \Omega_{S D}$ as follows:

$$
\omega_{S D}^{*}\left(t_{S}\right)=\underset{\omega \in \Omega_{S D}}{\arg \min }\left[\begin{array}{l}
T\left(\omega, t_{S}\right) \\
R\left(\omega, t_{S}\right) \\
N\left(\omega, t_{S}\right)
\end{array}\right]=\underset{\omega \in \Omega_{S D}}{\arg \min }\left[\begin{array}{c}
\sum_{e_{i} \in \omega} \operatorname{tr}\left(e_{i}, t\right) \\
\sum_{e_{i} \in \omega} r\left(e_{i}, t\right) \\
\sum_{e_{i} \in \omega} n\left(e_{i}, t\right)
\end{array}\right]
$$

subject to

$$
\operatorname{tr}\left(e_{i}, t\right) \geq 0 \quad r\left(e_{i}, t\right) \geq 0 \quad n\left(e_{i}, t\right) \geq 0
$$

where $T\left(\omega, t_{S}\right), R\left(\omega, t_{S}\right)$ and $N\left(\omega, t_{S}\right)$ represent the cumulative travel time, added resistance, and navigation risk, respectively, for the selected route/walk $\omega \in \Omega_{S D}$ with predefined departure time $t_{S}$. It is worth mentioning that the environmental conditions change over time and space, therefore even if the structure of the graph $\mathcal{G}=(\mathcal{N}, \mathcal{E})$ is kept constant in terms of connection edges (or waterways), the costs of each edge $(c(e, t))$ vary on time, as highlighted through the dependency of the time variable $t \in\left[t_{s}, t_{D}\right]$ ( $t_{D}$ representing the upper bound of the arrival time) of the selected costs. As output, the planning layer produces a subset of $\Omega_{S D}$ of the dominant walks in terms of the defined costs i.e., $\omega_{S D}^{*}\left(t_{S}\right)$ (as shown in Figure 1). Finally, this set is analyzed at the decision layer.

\subsection{Decision Layer}

The Decision layer represents the component of the proposed weather-routing system where the decision-maker interacts with the system to select the final route. In the proposed system, the planning 
layer produces a set of Pareto dominant solutions as a result of the multi-objective optimization problem characterized by the 3 conflicting criteria (costs). The introduction of the navigation safety rules in the form of minimization criterion allows deeper analysis of the Pareto front, compared to other methodologies [5].

The idea proposed in this paper is to group the Pareto solutions based on the value of the cumulative navigation risk, $N\left(\omega, t_{S}\right)$, associated with each route. To this aim, a set of navigation risk limits or thresholds must be empirically identified. These thresholds represent for how long the dangerous phenomena persist during the transit. In general, the more the IMO dangerous phenomena persist during the transit, the more the route will be characterized by high navigation risk. Based on these thresholds, the Pareto solutions are divided into disjoint sets. Then, for each set defined by different navigation risk thresholds or limits, the route representing the trade-off solution with respect to the remaining criteria (travel time $T\left(\omega, t_{S}\right)$, added resistance $R\left(\omega, t_{S}\right)$ ) is selected. This produces a subset of routes from the set of Pareto dominant solutions (i.e., one for each group defined by different navigation risk thresholds) as an output to the decision-maker. This analysis is produced and visualized through the HRV method [29].

Finally, the operator interacts with the system to select the route from the obtained subset representing the best trade-off based on his/her own navigation experience and additional requirements. This design choice allows a good understanding and comparison of the available solutions as discussed in the next section where the proposed system is tested in a real scenario.

\section{Results: Testing the Proposed System in a Real Scenario}

\subsection{Scenario Setup}

The proposed system is tested in the scenario of planning a route from La Spezia port (ITA) to Gibraltar port (UK) in the west part of the Mediterranean Sea. The selected AOI and time-frame provided through the Estimated Time of Departure (ETD) are:

- $\quad$ AOI longitude range: from $6.5^{\circ} \mathrm{W}$ to $10.5^{\circ} \mathrm{E}$.

- AOI latitude range: from $35.0^{\circ} \mathrm{N}$ to $45.0^{\circ} \mathrm{N}$.

- $\quad$ ETD: 10 October 2018 22:00 UTC.

The selected AOI and time-frame represents a good test case for evaluating the performance of the proposed system due to the presence of a storm crossing the AOI, as depicted in Figure 4 . The scenario considers the multi-role frigate defined in Table 1.

\subsection{Environmental Layer Setup}

In this scenario, the Environmental layer is interfaced with the data provider DICCA-MeteOcean [11,30] constantly access to newly updated forecasts. The forecasts are generated through the Wavewatch III model [31]. The forecasts are provided on a standard spatial grid of $10 \mathrm{~km}(5.39 \mathrm{nmi})$ resolution and $1 \mathrm{~h}$ temporal resolution. The forecast covers a temporal window of $120 \mathrm{~h}$ and is computed daily at 00:00 UTC. The uncertainty associated with the METOC variables is not available. In the selected scenario, recalling that ETD is scheduled for 10 October 2018 22:00 UTC, the most recent up to date forecast is used. This was computed 10 October 2018 00:00 UTC. The bathymetry/depth profiles are provided by the EMODNet Network [32] allowing the identification of the available routes and the areas where the navigation is not possible. For completeness, Table 2 reports the complete set of environmental variables retrieved from the selected providers in the proposed setup. As shown in the METOC snapshots in Figure 4 for the temporal window 10 October 2018 22:00 UTC-12 October 2018 16:00 UTC, the AOI is crossed by a storm characterized by $H_{S}>3 \mathrm{~m}$. This represents an environmental risk that may produce dangerous navigation conditions according to IMO [8]. 


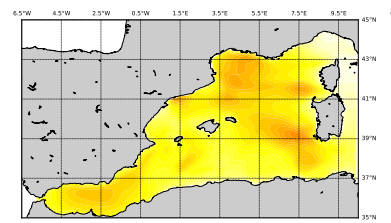

(a) 10-10-2018 22:00 UTC

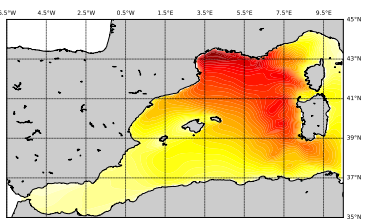

(e) 10-11-2018 22:00 UTC

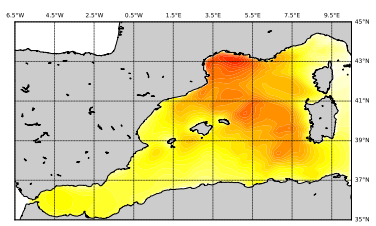

(b) 10-11-2018 04:00 UTC

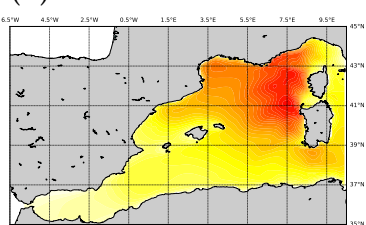

(f) 10-12-2018 04:00 UTC

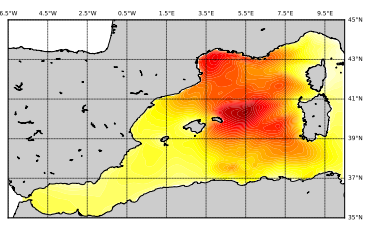

(c) 10-11-2018 10:00 UTC

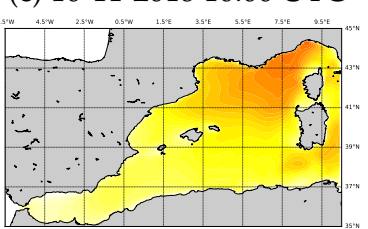

(g) 10-12-2018 10:00 UTC

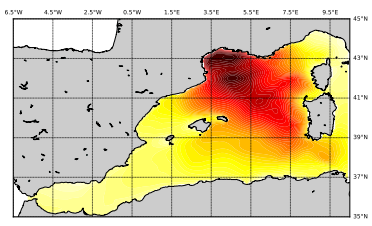

(d) 10-11-2018 16:00 UTC

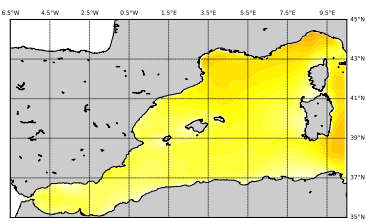

(h) 10-12-2018 16:00 UTC

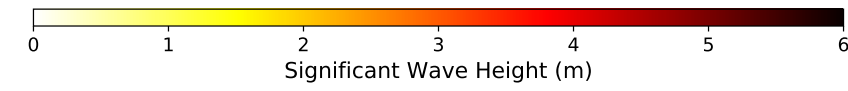

Figure 4. Evolution of the significant wave height $\left(H_{S}\right)$ in the AOI, with snapshots (Figures 14.a-14.h) every $6 \mathrm{~h}$ in the selected temporal window (10 October 2018 22:00 UTC-12 October 2018 16:00 UTC).

\subsection{Planning Layer Setup: Navigation Graph Construction}

The nominal route (provided by searoutes.com [24]) is used for the generation of the navigation graph as depicted in Figure 5.

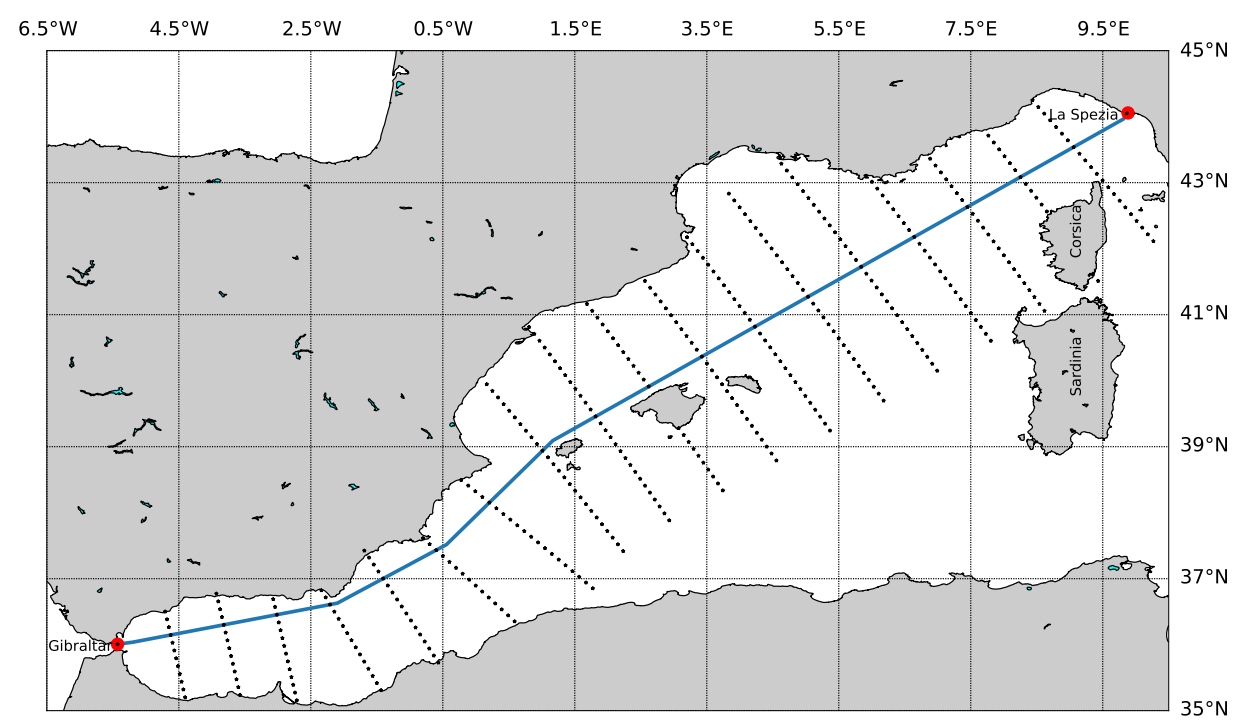

Figure 5. Nominal route between La Spezia (ITA) and Gibraltar (UK) ports provided by searoutes. com [24]. Navigation graph with nodes plotted as black point markers.

The route is plotted in blue color and the nodes composing the navigation graph as black point markers. In this scenario, the navigation graph is generated through $m=20$ stages. Each stage (perpendicular to the nominal route) measures $b=107.99 \mathrm{nmi}$ and collects $q=31$ equidistant nodes, presenting a stage spatial resolution of $\frac{b}{q}=3.48 \mathrm{nmi}$. After the removal of the edges and nodes where the navigation is not allowed (e.g., reduced maneuverability of the ship due to shallow water, etc.) the resulting navigation graph $\mathcal{G}=(\mathcal{N}, \mathcal{E})$ is characterized by 388 valid nodes and 7589 valid edges in total, with an average edge length of $79.91 \mathrm{nmi}$, as reported in Table 3. The nominal route represents the shortest seaway connection between departure and destination ports. 
Table 2. Environmental variables retrieved by the Environmental layer from the selected providers.

\begin{tabular}{lccc}
\hline Parameter & Symbol & Units & Data Provider \\
\hline Significant wave height & $H_{S}$ & $\mathrm{~m}$ & DICCA-MeteOcean [11] \\
Wave period & $T$ & $\mathrm{~s}$ & DICCA-MeteOcean [11] \\
Wavelength & $\lambda$ & $\mathrm{m}$ & Hunt's method [33] function of T and $d$. \\
Wave direction & $\alpha$ & ${ }^{\circ}$ respect to North & DICCA-MeteOcean [11] \\
\hline Wind speed & $U_{10}$ & $\mathrm{~m} \mathrm{~s}^{-1}$ & DICCA-MeteOcean [11] \\
Wind direction & $\phi$ & ${ }^{\circ}$ respect to North & DICCA-MeteOcean [11] \\
\hline Depth & $d$ & $\mathrm{~m}$ & EMODNet [32] \\
\hline
\end{tabular}

Table 3. Navigation graph main properties and associated values for the selected scenario.

\begin{tabular}{lccc}
\hline Property & Symbol & Value & Units \\
\hline Number of stages & $m$ & 20 & - \\
Stage length & $b$ & 107.99 & $\mathrm{nmi}$ \\
Number of nodes per stage & $q$ & 31 & - \\
Number of valid nodes & $|\mathcal{N}|$ & 388 & - \\
Number of valid edges & $|\mathcal{E}|$ & 7589 & - \\
Average edge length & & 79.91 & $\mathrm{nmi}$ \\
\hline
\end{tabular}

The spatial and temporal resolution of the data provided by the Environmental layer has a direct impact on the navigation graph, and consequently in the Planning layer. As matter of fact, the costs associated with each edge of the navigation graph are computed based on the average METOC values of the grid cells crossed by the ship sailing on the edge under consideration. This is depicted in Figure 6, where the costs of the edge $e$ connecting the node $i$ with $j$ are computed based on the grid cells touched by the edge $e$, highlighted in grey color. In the used setup, based on the navigation graph, each edge (average edge length $79.91 \mathrm{nmi}$ ) collects on average the METOC values of 14 grid cells (METOC model resolution $10 \mathrm{~km}(5.39 \mathrm{nmi}))$.

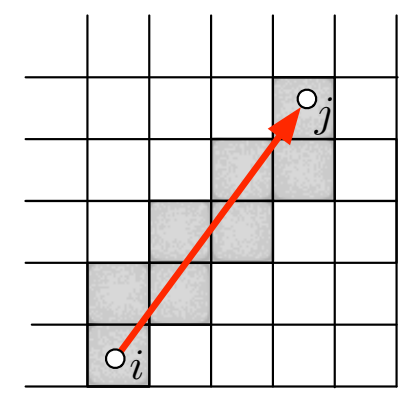

Figure 6. Schematic representation of the METOC grid cells touched by an edge (highlighted in grey color) connecting two nodes of the spatial graph grid.

In general, the navigation graph determines the domain of the available routes connecting the departure and destination ports, as well as the subset of the environmental data processed within the AOI. Due to the dynamic nature of the navigation graph (dependency of the edge costs with the time), it is required a trade-off analysis of the computation time, the size of the domain solutions and the ship operator requirements for the determination of the most convenient property values of the navigation graph. The configuration reached for this scenario is the result of various tests estimating the computational time to reach the final solution, based on the ship operator requirements. A deeper investigation of the size of the navigation graph will be addressed in future works. In the selected scenario the nominal travel time to transit from La Spezia port to Gibraltar port is $44 \mathrm{~h}$. 


\subsection{Output of Planning Layer}

The output produced by the Planning layer is depicted in Figure 7. It shows the set of Pareto efficient routes connecting the departure and destination ports. Each route represents a different trade-off among the selected criteria (or costs). The usage of the Martins algorithm [22] (at the Planning layer) allows the computation of the complete set of Pareto solutions and not a subset of the Pareto routes as provided by other solving methods (e.g., genetic algorithms [21]). Furthermore, the incorporation of the navigation risk in the proposed multi-criteria setup allows the estimation of the total risk associated with each Pareto optimal route.

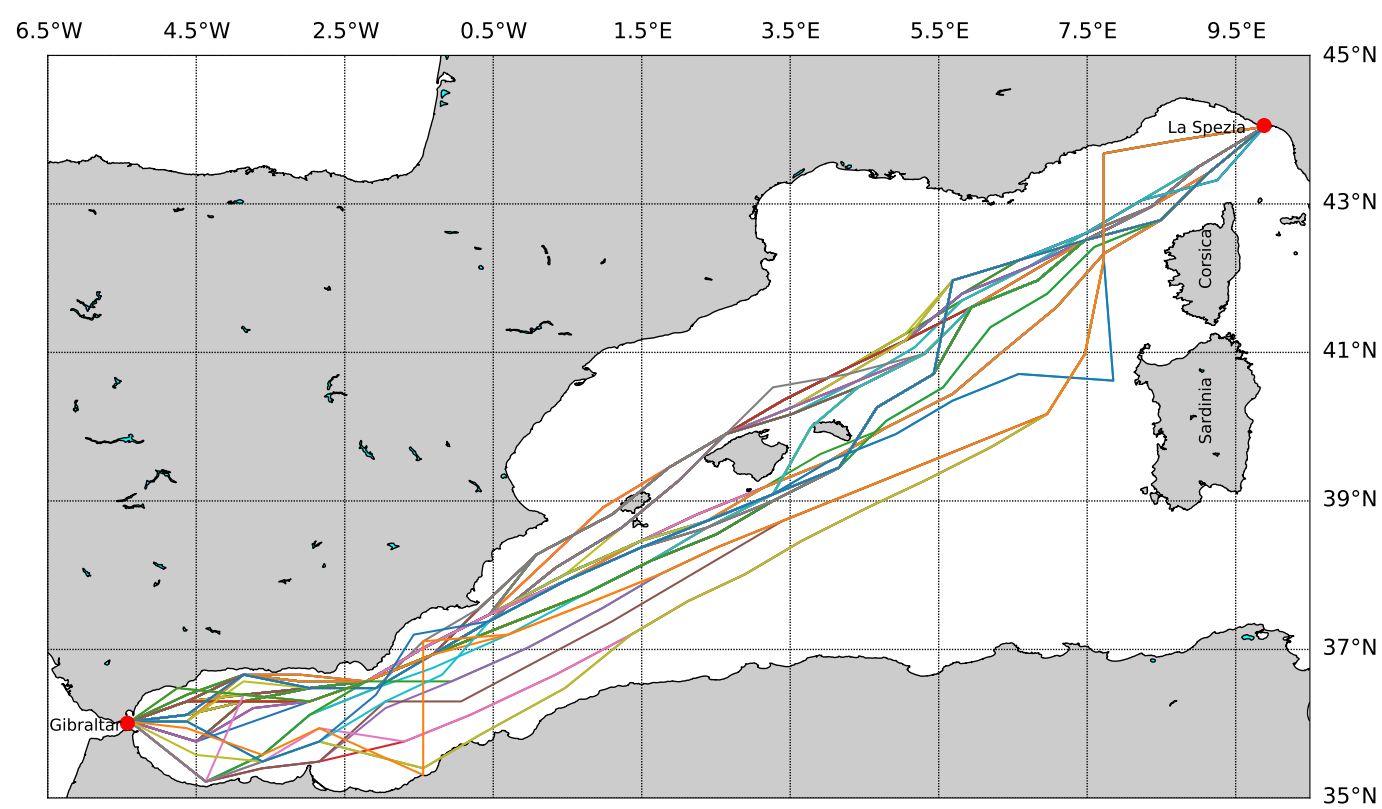

Figure 7. Set of Pareto efficient routes generated through the weather-routing process in the selected scenario.

Figure 8 allows deeper analysis of the set of Pareto solutions in a 4-dimensional plot, where each axis represents a different minimization criterion: (cost 1: $x$ axis—-travel time, cost $2: y$ axis—navigation added resistance and cost 3: $z$ axis-navigation risk). To simplify the analysis, the navigation risk is also associated with a color map (fourth dimension): the solutions in purple and blue colors are associated with the routes characterized by a low risk; the solutions in lighter colors (green, yellow) are characterized by medium to high risk.

An analysis of the results of Figure 8 shows that the solutions characterized by low travel time are the ones characterized by high navigation added resistance (therefore travel cost). The medium-high navigation risk routes are more concentrated in the group of the routes with low travel time. Instead, the low-risk routes span most of the Pareto frontier on the $x-y$ plane (travel time and navigation added resistance). Finally, the proposed system must identify the solution representing the candidate trade-off solution. This is identified through the Decision layer, which is provided in Section 3.5. 


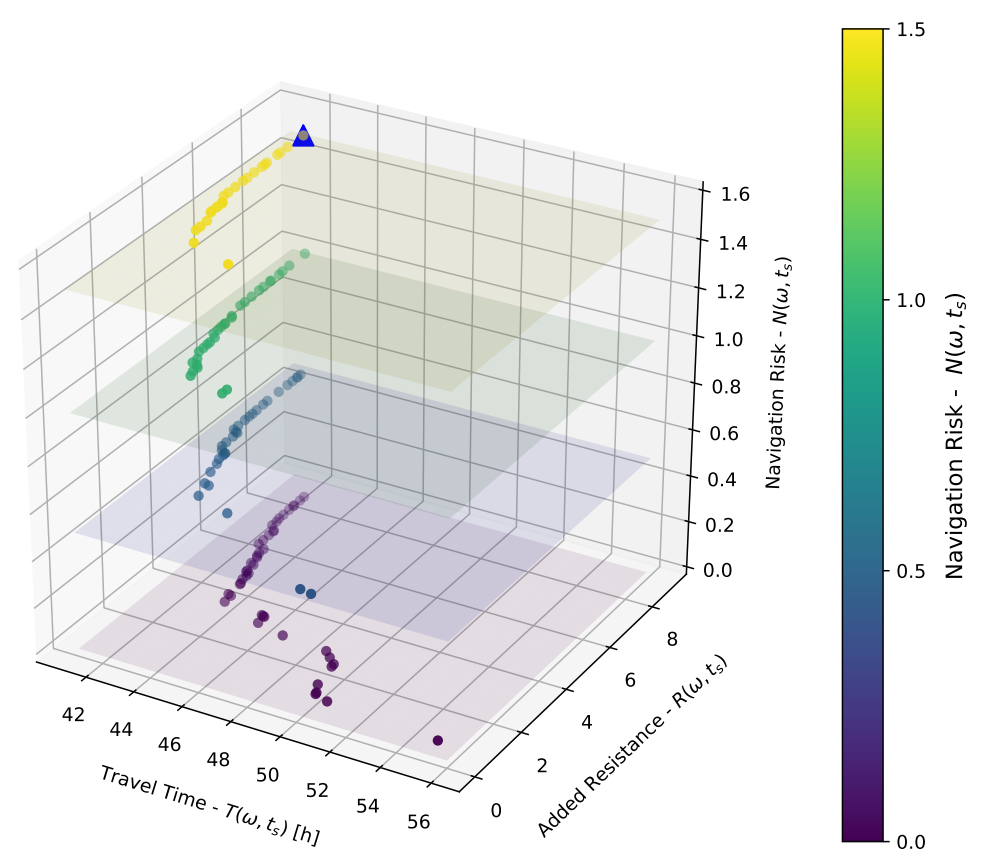

Figure 8. Set of Pareto efficient solutions at the output of the planning layer for the selected scenario. Each axis represents a different criterion: $x$ axis-travel time, $y$ axis-navigation added resistance and $z$ axis-navigation risk. The solutions are also grouped by navigation risk color, with dark (purple, blue) and light (green, yellow) colors for low and medium-high risks, respectively. The blue triangular marker identifies the costs associated with the nominal route [24].

One way to identify the route representing the best trade-off solution is through the HRV [29] methodology: One way to define a meaningful representation of the Pareto set is through the HRV [29]. According to this method to represent multiple objectives having different values, it is required to normalize each objective function. In this work, a linear normalization is applied. The 3 objectives are normalized as follows in Equation (12):

$$
\begin{aligned}
\bar{T}\left(\omega, t_{S}\right) & =\frac{T\left(\omega, t_{S}\right)}{\max _{\omega \in \omega^{*}\left(t_{S}\right)} T\left(\omega, t_{S}\right)} \\
\bar{R}\left(\omega, t_{S}\right) & =\frac{R\left(\omega, t_{S}\right)}{\max _{\omega \in \omega^{*}\left(t_{S}\right)} R\left(\omega, t_{S}\right)} \\
\bar{N}\left(\omega, t_{S}\right) & =\frac{N\left(\omega, t_{S}\right)}{\max _{\omega \in \omega^{*}\left(t_{S}\right)} N\left(\omega, t_{S}\right)}
\end{aligned}
$$

Figure 9 presents the Pareto solution set in a 3-dimension space, where: $x$ axis $=$ normalized travel time; $y$ axis = the normalized navigation added resistance; and the color domain represents the routes by the normalized risk. As above-mentioned, the values in this representation are now associated with the range $[0,1]$. The most desirable solution from the Pareto set is identified by evaluating the distance of each solution from the Utopia point (point $(0,0,0)$ - the hypothetical point that corresponds to the minimum value of each individual criteria [34]). This allows the navigator to have a clear and intuitive reference-point during the decision-making process. It is worth pointing out that the front of low-risk routes (purple color) are characterized by higher travel time $\left(T\left(\omega, l_{S}\right)\right)$ for a given added resistance value $\left(R\left(\omega, l_{S}\right)\right)$ than for the high-risk routes (green, yellow routes). This fact suggests that the routes with low travel time are the ones with high risk of the scenario under study. Following the HRV methodology [29], the route representing the best trade-off (the closest solution to the Utopia point) is depicted with a red triangular marker in Figure 9. 


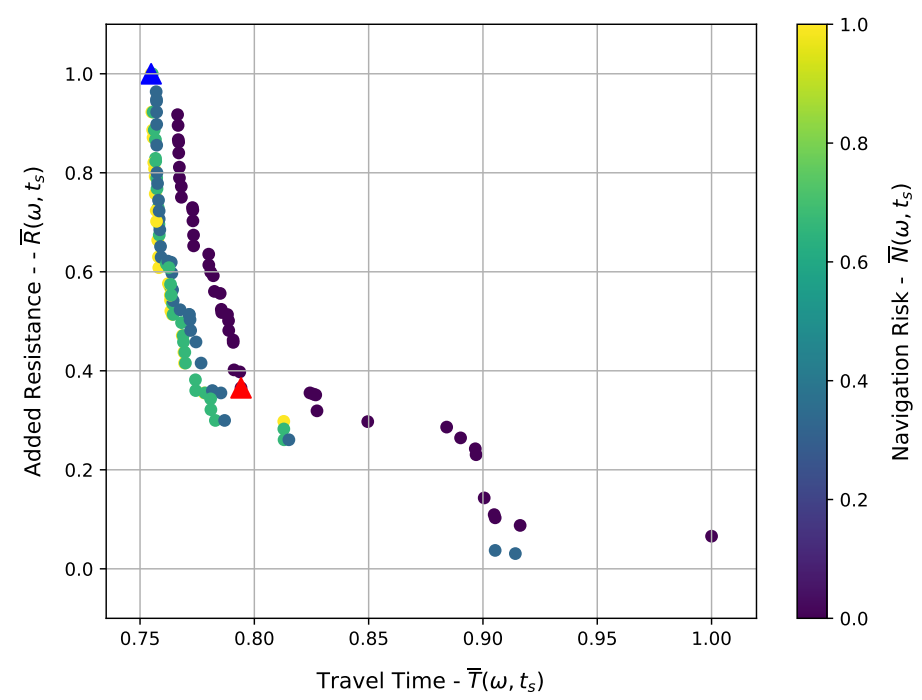

Figure 9. Set of Pareto efficient solutions at the output of the planning layer for the selected scenario. The solutions are plotted in the 3-dimension space: $x$ axis-travel time, $y$ axis-navigation added resistance and the color domain to identify the routes by risk. The red triangular marker identifies the route representing the best trade-off solution respect to the Utopia point. The blue triangular marker identifies the costs associated with the nominal route [24].

\subsection{Output of Decision Layer}

The decision layer performs the important task of extrapolating the most informative data from the output of the planning layer $\left(\omega_{S D}^{*}\right)$, to drive the operator in the selection of the final route. This is achieved through the identification of a subset of the Pareto dominant solutions with ad hoc properties. This design choice improves the situational awareness of the scenario and facilitates the interaction of the operator with the system.

The decision layer exploits the navigation risk criterion to group the routes characterized by cumulative navigation risk $N\left(\omega, t_{S}\right)$ within the same range of values, or threshold $\delta_{N}$. Consequently, the routes are grouped in parallel $x-y$ planes with different $z$ navigation risk thresholds, $\delta_{N}$, as depicted in Figure 8. Then, for each navigation risk threshold $\delta_{N}$ (or $x-y$ plane), the route representing the candidate trade-off solution with respect to the remaining criteria (travel time $T\left(\omega, l_{S}\right)$ ), added resistance $\left.R\left(\omega, l_{S}\right)\right)$ is selected. For the selected scenario, the 4 levels of navigation risk are identified as from Equation (13). The 4 levels specify the closeness and the persistence of dangerous phenomena for the selected transit. More precisely, the high risk level $\left(N\left(\omega, t_{S}\right) \geq 1.25\right)$ means that for more than one edge $e \in \omega$ the dangerous phenomena persists; in the medium-high risk level $\left(0.75 \leq N\left(\omega, t_{S}\right)<1.25\right)$ the dangerous phenomena persist at least for one edge $e \in \omega$ or the route is characterized by protracted closeness to dangerous phenomena. In the medium-low risk level $\left(0.25 \leq N\left(\omega, t_{S}\right)<0.75\right)$ at least one edge is characterized by protracted closeness to the dangerous phenomena. Finally, in the low-risk level $\left(0 \leq N\left(\omega, t_{S}\right)<0.25\right)$ the route is safe. The routes associated with each navigation risk threshold $\delta_{N}$ and its cumulative cost values are reported in Table 4. For completeness of the analysis, the produced routes are compared with the nominal route (used for the generation of the navigation graph) [24].

$$
\delta_{N}= \begin{cases}\text { High risk } & N\left(\omega, t_{S}\right) \geq 1.25 \\ \text { Medium-High risk } & 0.75 \leq N\left(\omega, t_{S}\right)<1.25 \\ \text { Medium-Low risk } & 0.25 \leq N\left(\omega, t_{S}\right)<0.75 \\ \text { Low risk } & 0 \leq N\left(\omega, t_{S}\right)<0.25\end{cases}
$$

The nominal (ID = 0) and selected routes (IDs $=1,2,3,4)$ are depicted in Figure 10. The routes identified by high navigation risk $\left(N\left(\omega, l_{S}\right)=1.5\right)$ plotted in blue $(\mathrm{ID}=0)$ and orange $(\mathrm{ID}=1)$ 
colors, including the nominal route, are the ones pushed in the northern side of the Balearic Islands, which provide lower travel-times. Furthermore, the nominal route (ID $=0$, in blue) presents a considerable high added resistance (and consequential travel cost) for traveling on the shortest route toward the destination port due to the adverse weather conditions in the northern side of the Balearic Islands.

Table 4. Costs value for the nominal route $(\mathrm{ID}=0)$ and the trade-off routes (IDs $=1-4)$.

\begin{tabular}{cccc}
\hline ID & Travel Time $\boldsymbol{T}\left(\boldsymbol{\omega}, \boldsymbol{t}_{\boldsymbol{S}}\right)[\mathrm{h}]$ & Added Resistance $\boldsymbol{R}\left(\boldsymbol{\omega}, \boldsymbol{t}_{\boldsymbol{S}}\right)$ & Navigation Risk $\boldsymbol{N}\left(\boldsymbol{\omega}, \boldsymbol{t}_{\boldsymbol{S}}\right)$ \\
\hline 0 & 41.52 & 8.92 & 1.5 \\
1 & 43.08 & 2.81 & 1.5 \\
2 & 43.13 & 2.67 & 1.0 \\
3 & 43.35 & 2.67 & 0.5 \\
4 & 43.75 & 3.26 & 0.0 \\
\hline
\end{tabular}

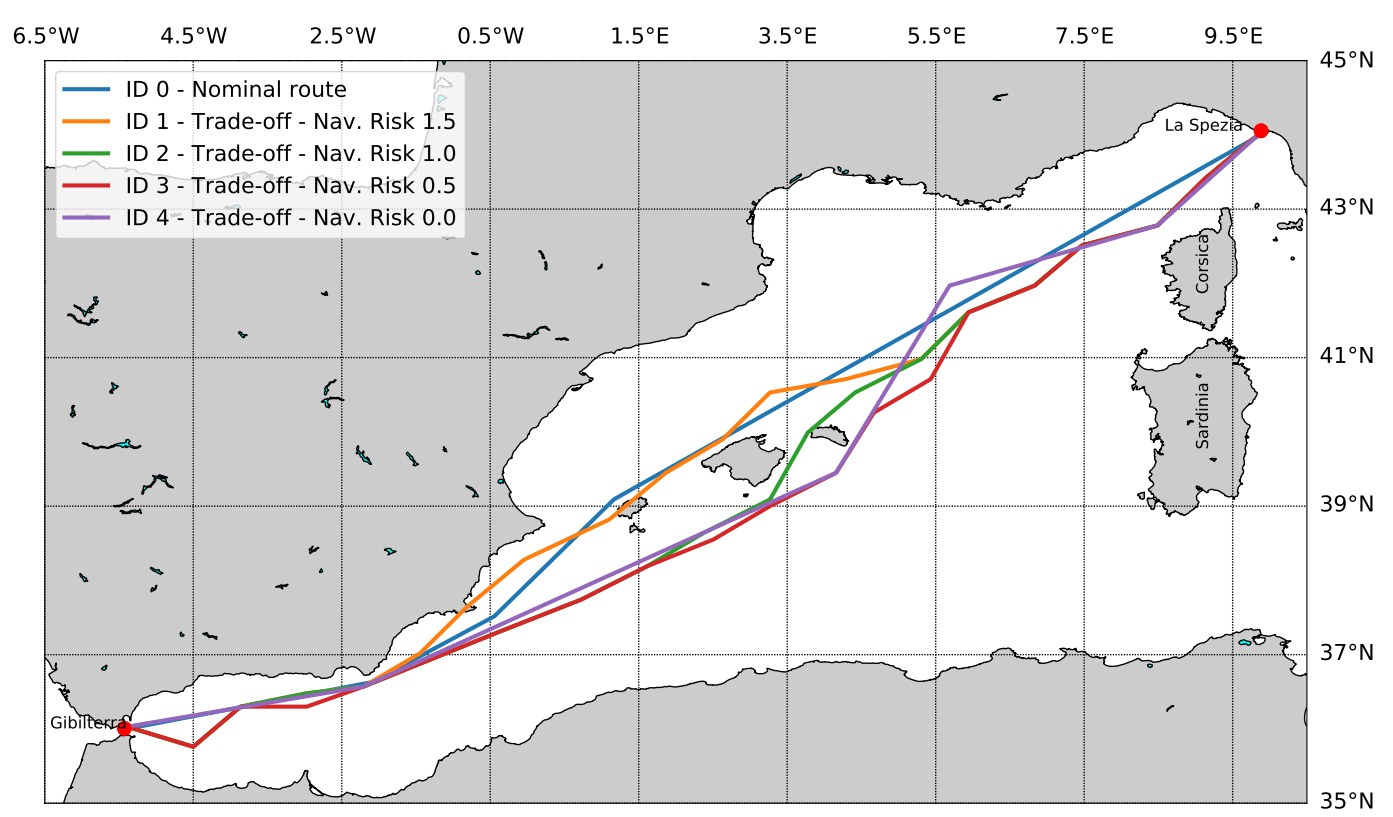

Figure 10. Set of Pareto routes representing the candidate trade-off solution for each navigation risk threshold compared with the nominal route in blue color (provided by the web-service searoutes. com [24]).

The remaining 3 routes, ID $=2,3,4$, are pushed to the southern side of the Balearic Islands. This maneuver moves the ship away from the perturbation affecting the coastlines of France and Italy $\left(H_{S} \geq 3 \mathrm{~m}\right)$ in the time window under analysis (see Figure 4). This provides safer navigation conditions $\left(N\left(\omega, t_{S}\right)<1.5\right)$. At this stage, the operator can select the final route from the provided subset. This process not only considers the evaluation of the different trade-offs associated with each route, but also considers the gained experience of the operator, as well as any other additional requirements. The data provided in Table 4 suggests as output route the one characterized by the lowest navigation risk (ID = 4, purple color in Figure 10). As matter of fact, this route presents values in terms of travel time comparable to the ones of the nominal route, but with a reduced added resistance and with minimum navigation risks that may cause damages to the cargo or dangerous situations for the ship crew.

\section{Conclusions and Future Work}

A weather-routing system based on a multi-criteria optimization setup characterized by a set of 3 conflicting criteria (travel time, ship navigation added resistance and navigation safety/risk) is presented in this paper. The proposed system supports decision-making while planning navigation 
routes by providing to the decision-makers a set of optimal routes with different criteria instead of one optimal route. This allows operators making their decisions for route planning based on their personal criteria and experience.

The main contributions of the paper are four. First, the proposed weather-routing system can identify the routes characterized by safe METOC conditions from a navigation point of view. This is supported by the results obtained for the real scenario under study. This allows mitigating dangerous sailing conditions. Second, the exploitation of the IMO guidelines [8] in the form of optimization criterion allows the identification of the best trade-off route for a desired navigation risk threshold. Third, it is demonstrated how the proposed system facilitates the interaction between the operator and the system at the decision layer. Instead of providing just one route to the operator (representing the optimal trade-off combining the 3 criteria), the reduced set of trade-off routes ranked by navigation risk allows a deeper comparison and understanding of the scenario. And fourth, the set of proposed routes are all characterized by a 3-5\% of increase in the travel time and route length compared to the nominal route. At the same time, the navigation risk is reduced up to almost no risk of navigation in one of the proposed solutions.

Future developments could be focused on the increase of the computational performance of the weather-routing process. This could be done by introducing a novel navigation graph based on the partitioning of the AOI in convex sections, characterized by uniform weather and risk conditions. This allows the reduction of the size of the navigation graph and consequently a reduction of the computation time, providing proposed solutions in a faster way.

Author Contributions: Conceptualisation: R.V.-B. and T.F.; Methodology: T.F. and R.V.-B.; Software: T.F.; Validation: T.F. and R.V.-B.; Formal analysis: T.F. and R.V.-B.; Investigation: T.F. and R.V.-B.; Resources: R.V.-B.; Data curation: T.F.; Writing-original draft preparation: T.F.; Writing-review and editing: R.V.-B.; Visualisation: T.F.; Supervision: R.V.-B.; Project administration: R.V.-B.; Funding acquisition: R.V.-B.

Funding: This work has been funded by the Defence Research and Development Canada (DRDC) under the project "Mission Planning Aid-Decision Support and Risk Assessment" (MPA-DeSRA). The project has been developed in collaboration with the Environmental Knowledge and Operational Effectiveness/Maritime Intelligence Surveillance and Reconnaissance (EKOE/MISR) programme at the NATO STO Centre for Maritime Research and Experimentation (CMRE).

Acknowledgments: The authors thank also the DICCA-MeteOcean Department [11] for providing the METOC forecast data for the scenario.

Conflicts of Interest: The authors declare no conflict of interest with any of the parts involved in the research work presented in this paper and its publication. This includes explicit authorisation from the funder to publish the research work presented here (concept, algorithms, development, results and conclusions).

\section{References}

1. Perera, L.P.; Soares, C.G. Weather routing and safe ship handling in the future of shipping. Ocean Eng. 2017, 130, 684-695. [CrossRef]

2. Zwierzewicz, Z. Algorithms for the Ship Trajectory Planning and Its Tracking in the Collision A voidance Process. IFAC Proc. Vol. 2001, 34, 523-529. [CrossRef]

3. Mannarini, G.; Pinardi, N.; Coppini, G.; Oddo, P.; Iafrati, A. VISIR-I: Small vessels, least-time nautical routes using wave forecasts. Geosci. Model Dev. 2015, 8, 7911-7981. [CrossRef]

4. Sidoti, D.; Avvari, G.V.; Mishra, M.; Zhang, L.; Nadella, B.K.; Peak, J.E.; Hansen, J.A.; Pattipati, K.R. A Multiobjective Path-Planning Algorithm with Time Windows for Asset Routing in a Dynamic Weather-Impacted Environment. IEEE Trans. Syst. Man Cybern. Syst. 2017, 47, 3256-3271. [CrossRef]

5. Fabbri, T.; Vicen-Bueno, R.; Grasso, R.; Pallotta, G.; Millefiori, L.M.; Cazzanti, L. Optimization of surveillance vessel network planning in maritime command and control systems by fusing METOC and AIS vessel traffic information. In Proceedings of the OCEANS 2015-Genova, Genoa, Italy, 18-21 May 2015; pp. 1-7. [CrossRef]

6. Fang, M.C.; Lin, Y.H. The optimization of ship weather-routing algorithm based on the composite influence of multi-dynamic elements (II): Optimized routings. Appl. Ocean Res. 2015, 50, 130-140. [CrossRef] 
7. Krata, P.; Szlapczynska, J. Ship weather routing optimization with dynamic constraints based on reliable synchronous roll prediction. Ocean Eng. 2018, 150, 124-137. [CrossRef]

8. IMO. Revised Guidance To The Master For Avoiding Dangerous Situations In Adverse Weather And Sea Conditions; Technical Report; International Maritime Organization (IMO): London, UK, 2007.

9. Krata, P.; Szlapczynska, J. Weather Hazard Avoidance in Modeling Safety of Motor-Driven Ship for Multicriteria Weather Routing. TransNav Int. J. Mar. Navig. Saf. Sea Transp. 2012, 6, 71-78. [CrossRef]

10. Szlapczynski, R.; Krata, P. Determining and visualizing safe motion parameters of a ship navigating in severe weather conditions. Ocean Eng. 2018, 158, 263-274. [CrossRef]

11. Dipartimento di Ingegneria Civile, Chimica e Ambientale (DICCA). MeteOcean-DICCA. 2018. Available online: http:/ / www.dicca.unige.it/meteocean/ (accessed on 1 June 2018).

12. Thompson, I. Fatigue damage variation within a class of naval ships. Ocean Eng. 2018, 165, 123-130. [CrossRef]

13. Vantorre, M.; Eloot, K.; Delefortrie, G.; Lataire, E.; Candries, M.; Verwilligen, J. Maneuvering in Shallow and Confined Water. In Encyclopedia of Maritime and Offshore Engineering; American Cancer Society: Atlanta, GA, USA, 2017; pp. 1-17. [CrossRef]

14. U.S. Army Engineer Waterways Experiment Station, Coastal Engineering Research Center. Direct Methods for Calculating Wavelength; Coastal Engineering Technical Note CETN-1-17; U.S. Army Engineer Waterways Experiment Station: Vicksburg, MS, USA, 1985.

15. Perez, T. Ship Motion Control: Course Keeping and Roll Stabilisation Using Rudder and Fins, 1st ed.; Springer Publishing Company: London, UK, 2010.

16. Lin, Y.H.; Fang, M.C.; Yeung, R.W. The optimization of ship weather-routing algorithm based on the composite influence of multi-dynamic elements. Appl. Ocean Res. 2013, 43, 184-194. [CrossRef]

17. Bijlsma, S. Minimal Time Route Computation for Ships with Pre-Specified Voyage Fuel Consumption. J. Navig. 2008, 61, 723-733. [CrossRef]

18. Shao, W.; Zhou, P.; Thong, S.K. Development of a novel forward dynamic programming method for weather routing. J. Mar. Sci. Technol. 2012, 17, 239-251. [CrossRef]

19. Zyczkowski, M.; Szlapczynski, R. Multi-Objective Weather Routing of Sailing Vessels. Pol. Marit. Res. 2017, 24. [CrossRef]

20. Padhy, C.; Sen, D.; Bhaskaran, P. Application of wave model for weather routing of ships in the North Indian Ocean. Nat. Hazards 2007, 44, 373-385. [CrossRef]

21. Walther, L.; Rizvanolli, A.; Wendebourg, M.; Jahn, C. Modeling and Optimization Algorithms in Ship Weather Routing. Int. J. E Navig. Marit. Econ. 2016, 4, 31-45. [CrossRef]

22. Martins, E.Q.V. On a multicriteria shortest path problem. Eur. J. Oper. Res. 1984, 16, 236-245. [CrossRef]

23. Dijkstra, E.W. A Note on Two Problems in Connexion with Graphs. Numer. Math. 1959, 1, $269-271$. [CrossRef]

24. Maritime Data Systems. Searoutes.com. 2018. Available online: https://www.searoutes.com (accessed on 1 June 2018.)

25. Loeches, J.; Vicen-Bueno, R.; Mentaschi, L. METOC-driven Vessel Interdiction System (MVIS): Supporting decision making in Command and Control (C2) systems. In Proceedings of the OCEANS 2015-Genova, Genoa, Italy, 18-21 May 2015; p. 1-6.

26. Zyczkowski, M.; Krata, P.; Szlapczynski, R. Multi-Objective Weather Routing of Sailboats Considering Wave Resistance. Pol. Marit. Res. 2018, 25, 4-12. [CrossRef]

27. Lu, R.; Turan, O.; Boulougouris, E.; Banks, C.; Incecik, A. A semi-empirical ship operational performance prediction model for voyage optimization towards energy efficient shipping. Ocean Eng. 2015, 110, 18-28. [CrossRef]

28. West, D.B. Introduction to Graph Theory, 2 ed.; Prentice Hall: Upper Saddle River, NJ, USA, 2000.

29. Chiu, P.W.; Bloebaum, C.L. Hyper-Radial Visualization (HRV) method with range-based preferences for multi-objective decision making. Struct. Multidiscip. Optim. 2009, 40, 97. [CrossRef]

30. Mentaschi, L.; Besio, G.; Cassola, F.; Mazzino, A. Developing and validating a forecast/hindcast system for the Mediterranean Sea. J. Coast. Res. 2013, 2, 1551-1556. [CrossRef]

31. National Weather Service-Environmental Modeling Center. Wavewatch III Model. 2018. Available online: http:/ / polar.ncep.noaa.gov / waves/wavewatch/ (accessed on 1 June 2018). 
32. EMODnet Bathymetry-The European Marine Observation And Data Network. EMODnet—Understading the Topography of the European Seas. 2018. Available online: http://www.emodnet-bathymetry.eu/ (accessed on 1 June 2018).

33. Hunt, J.N. Direct solution of wave dispersion equation. J. Waterw. Port Coast. Ocean Eng. 1979, WW4, 457-459.

34. Miettinen, K. Nonlinear Multiobjective Optimization; Kluwer Academic Publishers: Boston, MA, USA, 1999.

(C) 2019 by the authors. Licensee MDPI, Basel, Switzerland. This article is an open access article distributed under the terms and conditions of the Creative Commons Attribution (CC BY) license (http://creativecommons.org/licenses/by/4.0/). 